\title{
Clinical Results of Platelet-Rich Plasma Injection in the Treatment of Androgenetic Alopecia
}

\author{
(D) Kadri Özer ${ }^{1}$, (1) Özlem Çolak² \\ ${ }^{1}$ Aydın State Hospital, Plastic, Reconstructive and Aesthetic Surgery, Aydın, Turkey \\ 2University of Health Sciences, İstanbul Okmeydanı Training and Research Hospital, Department of Plastic Surgery, Istanbul, Turkey
}

\section{Abstract}

Introduction: Platelet-rich plasma (PRP) represents a small volume of plasma in which the platelet concentration in normal blood is increased to optimum values. Its effect depends on the growth factors released from platelets. Although it has been investigated and used in many areas of plastic surgery, PRP injection has recently attracted interest in the treatment of hair loss. In this study, we aimed to evaluate the efficacy of adjuvant therapy in patients with male and female alopecia by establishing a standard protocol for the preparation and application of PRP.

Methods: Fifteen patients with mild to moderate androgenetic alopecia (Ludwig alopecia score I-II for women and Hamilton-Norwood score 1-4 for men) who had not received topical or systemic treatment for alopecia in the last 6 months were included in the study. Eleven of the patients were male and 4 were female. The mean age was 41 years (27-55). PRP was prepared using a single spin method with high acceleration force and low centrifugation time, and three sessions were performed with an interval of 3-4 weeks. Patient satisfaction was assessed by visual analogue scale (VAS). The assessments on day $0,1^{\text {st }}$ month, $2^{\text {nd }}$ month and $6^{\text {th }}$ month were evaluated retrospectively.

Results: Hair pull test was positive in all patients (100\%) before the treatment and the mean number of hair loss was 6.7. After the third treatment session, the hair pull test was negative in 13 patients (86.6\%) with a mean of number of 2.5 hair loss. An improvement in hair density and quality was demonstrated with macroscopic photographs taken in a standard manner before and after the procedure. The mean VAS value decreased from 6.8 to 2.1 after the procedure.

Discussion: PRP injections, which are prepared in a simple way without using a commercial kit, are an effective treatment option in alopecia treatment with high patient satisfaction and low cost.

Keywords: Alopecia, androgenetic alopecia, platelet, platelet-rich plasma

\section{INTRODUCTION}

Alopecia is divided into two main groups as cicatricial and noncicatricial. Androgenetic alopecia (AGA) is in the non-cicatricial group and is the most common type of hair loss in both men and women $(1,2)$. It is an inherited, androgen-dependent disorder characterized by progressive hair loss. However, genetic predisposition is very important to better determine the onset, severity and form of the disease. The hair follicles in the affected scalp area undergo a gradual transformation that causes shrinkage (miniaturization) due to the shortened anagen phase.
With each hair cycle, anagen becomes shorter and telogen remains constant $(2,3)$. As it is thought that hair is an important feature in the self-image of the person, the quality of life of patients diagnosed with AGA may be affected and it may cause emotional stress and decrease in self-esteem $(3,4)$.

Drug therapies approved by the US Food and Drug Administration (FDA) for the treatment of androgenetic alopecia are limited to topical minoxidil and oral finasteride. They can be used in single or combined form. These treatments have limited efficacy, and they also have topical and systemic side effects. Therefore, 
effective treatment modalities with fewer side effects were investigated. Recently, new approaches such as the application of platelet-rich plasma (PRP) have been introduced and some studies have shown promising results $(3,5)$.

PRP represents the autologous concentration of human platelets in a small plasma volume, in which the normal blood platelet concentration is optimized (at a minimum of 2 times baseline concentration to a maximum of 4 to 6 times baseline concentration). When platelet alpha granules are activated, they secrete numerous growth factors (GFs) that stimulate cell proliferation, differentiation and angiogenesis. The regenerative potential of PRP depends on these GFs. GFs act on the convex part of the follicle to which they are attached to their respective receptors in the stem cells, thus stimulating neovascularization by stimulating new follicle development (4-6).

Although there is no general consensus about the preparation, dosage, frequency and route of administration for PRP, there is also no reliable method for evaluating the results. The aim of this study was to establish a standardized protocol for the preparation and application of PRP in patients diagnosed with male and female AGA and to evaluate the effectiveness of the treatment.

\section{METHODS}

This study was approved by the Ethics Committee of University of Health Sciences, Ankara Training and Research Hospital Hospital and informed consent forms were signed by all patients. Fifteen patients who were followed-up for AGA since January 2016 and who had at least six months follow-up after three doses of PRP were included in the study. The assessments on day $0,1^{\text {st }}$ month, $2^{\text {nd }}$ month and $6^{\text {th }}$ month were evaluated retrospectively. All patients were in good general health and had mild to moderate AGA (Ludwig alopecia score I-II for women and Hamilton-Norwood score 1-4 for men). Eleven patients were male and four were female, and the mean age of patients over 18 years was 41 (27-55) years (Table 1). Patients who received topical or systemic AGA treatment in the last 6 months, those who had immunodeficiency due to malignancy, chemotherapy, steroid treatment, those who had dermatological diseases that would affect scalp, pregnant or nursing women, patients with autoimmune disease and hematological disease or those receiving anticoagulation treatment were not included in the study. Patients were instructed not to wash their hairs up to 2 days before the treatment and to stop smoking 2 weeks ago. Before the procedure, following conditions were ruled out by following tests: a) anemia by complete blood count, serum iron, serum ferritin and total iron binding capacity, folic acid and Vitamin B12, b) thyroid dysfunction by free T3, free T4 and TSH, c) syphilis by venereal disease research laboratory, d) polycystic ovarian syndrome by dehydroepiandrosterone sulfate, testosterone, androstenedione, prolactin, follicle-stimulating hormone and luteinizing hormone.

\section{Platelet-rich Plasma Protocol}

Approximately $11 \mathrm{~mL}$ of whole blood was added to 4 acid citrate dextrose-A (ACD-A) tubes in a ratio of 1:9. They were centrifuged at $1650 \mathrm{~g}$ for $5 \mathrm{~min}$ at room temperature. After centrifugation, the upper $1 / 3^{\text {rd }}$ of the supernatant (platelet-poor plasma) and the middle layer were transferred and collected in different injectors. The remaining lower $1 / 3^{\text {rd }}$ plasma (about $1 \mathrm{~mL}$ ) and the buffy-coat portion with precipitated platelets were obtained as PRP. Thus, a total of 5-6 mL of effective PRP from $45 \mathrm{~mL}$ of whole blood was transferred to $1 \mathrm{~mL}$ of insulin injectors.

PRP infiltration to the affected scalp area was performed using Nappage technique $\left(0.1 \mathrm{cc} / \mathrm{cm}^{2}\right.$, multiple small injection in linear pattern at intervals of $1 \mathrm{~cm}$ ) with $4 \mathrm{~mm}$ mesotherapy needles. Each patient received PRP at a total of 3 doses with an interval of 3-4 weeks. All patients were called for control at one-week intervals.

All patients underwent hair pull test at Day $0,1^{\text {st }}$ month, $2^{\text {nd }}$ month and $6^{\text {th }}$ month and results were noted (T1, pre-treatment; T2, $1^{\text {st }}$ month; T3, 2nd month; T4, $6^{\text {th }}$ month). Hair pull test was done by the same physician, by pulling a small amount of hair (approx. 100 pieces at the same time), and the number of hair loss along with the area of hair loss (to pull from the same area in the control) were recorded. The normal value is 1 to 3 hairs. More than 3 are considered positive. Anterior, lateral, posterior and vertex images were photographed in each evaluation to determine hair growth, volume, quality and fullness. Patients were asked to evaluate satisfaction on a $10 \mathrm{~cm}$ visual analog scale (VAS) before and 6 months after the procedure. This scale includes scales with facial images rated from 0 to 10.0 demonstrates maximum satisfaction and 10 demonstrates the worst satisfaction (Figure 1).

\section{Statistical Analysis}

SPSS version 20.0 (SPSS, Chicago, IL, USA) was used for data analysis. Kolmogorov-Smirnov test was used to assess the normality of the data. Paired t-test was used for normal distribution and Wilcoxon signed rank test was used for nonnormal distribution. 


\section{RESULTS}

Fifteen patients, who completed post-procedure $6^{\text {th }}$ month evaluation, were analyzed retrospectively. Three patients had a history of topical 5\% minoxidil lotion use due to hair loss, but no drug use was present within the 6 month period prior to PRP treatment.

Before the treatment, the hair pull test was positive in all patients (100\%) and the mean number of hair loss was 6.7 \pm 2.1 . After three sessions, the hair pull test was negative in 13 patients (86.6\%) and the mean number of hair loss was $1.6 \pm 1.5(p<0.0001)$. Patients noted a significant decrease in hair loss between the first and third injection (Figure 2). There was a general improvement in the hair density and quality that was demonstrated by the photos of the alopecic region taken from four different aspects at equal distance before and after the procedure (Figures 3, 4).

Patients were evaluated in terms of satisfaction on VAS before and 6 months after the procedure. While the mean pre-procedure VAS satisfaction score was $6.9 \pm 1.8$, this value was found to decrease to $2.1 \pm 1.4$ after the procedure $(p<0.0001)$. Table 1 shows the hair pull test and satisfaction scores of all patients before and 6 months after the treatment. All patients reported improvement in hair quality and thickness after the procedure, and 70\% reported an increase in hair density. Patients had no other side effects except spot bleeding, mild pain, and rash. There was no ecchymosis, infection or increase in hair loss in any patient.

\section{DISCUSSION}

Androgenetic alopecia is the most common hair disorder without satisfactory treatment and has a significant impact on psychological stress and is associated with low self-esteem. Current therapeutic strategies in the treatment of androgenetic alopecia, which is characterized by shorter anagen phase and shrunken hair follicles in the follicular unit, aim cellular proliferation and differentiation during the hair cycle (7).

The follicles in the scalp are found as a composite follicular unit. These composite follicular units contain primary follicles and several secondary follicles. Miniaturization starts first in secondary follicles. Initially, arrector pili muscle lost its connection with secondary follicles regressed in several follicular units. At this stage, the muscle is still attached to the primary follicle. The miniaturization continues progressively, and the connection between the muscle and the secondary follicles is lost. This leads to a reduction in hair density. Then the primary follicles are also affected from miniaturization and their connection with muscle is finally lost. Baldness occurs when all follicular units are miniaturized. The same miniaturization pattern and loss of muscle connection progressively continue until all follicular units are affected (8-10).

Androgenetic alopecia is a disorder associated with hormonal and genetic factors, and the treatment is mainly focused on hormonal blockade because the latter cannot be altered.

\begin{tabular}{|c|c|c|c|c|c|c|c|c|}
\hline Case & Gender & Age & $\begin{array}{l}\text { Alopecia } \\
\text { stage }\end{array}$ & $\begin{array}{l}\text { VAS score } \\
\text { (pre-procedure) }\end{array}$ & $\begin{array}{l}\text { VAS score } \\
\text { (post-procedure) }\end{array}$ & $\begin{array}{l}\text { Hair pull test } \\
\text { (pre-procedure) }\end{array}$ & $\begin{array}{l}\text { Hair pull test } \\
\text { (post-procedure) }\end{array}$ & $\begin{array}{l}\text { Previous } \\
\text { Treatment }\end{array}$ \\
\hline 2 & M & 42 & III & 8 & 4 & 10 & 3 & 5\% Minoxidil \\
\hline 3 & M & 27 & II & 6 & 2 & 4 & 0 & - \\
\hline 5 & M & 36 & 1 & 4 & 2 & 4 & 0 & - \\
\hline 6 & $\mathrm{~F}$ & 43 & II & 6 & 4 & 9 & 4 & - \\
\hline 7 & M & 31 & IV & 8 & 2 & 10 & 3 & 5\% Minoxidil \\
\hline 8 & M & 28 & II & 6 & 2 & 7 & 1 & - \\
\hline 12 & $\mathrm{~F}$ & 51 & II & 6 & 2 & 5 & 1 & - \\
\hline 13 & M & 44 & II & 8 & 0 & 6 & 0 & - \\
\hline 14 & M & 52 & II & 6 & 0 & 5 & 1 & - \\
\hline 15 & M & 55 & IV & 10 & 4 & 9 & 5 & - \\
\hline
\end{tabular}


Increased dihydrotestosterone (DHT), androgen receptors and caspase levels cause cellular apoptosis and hair loss (11). DHT plays a role in the inhibition of adenylyl cyclase (AMP-c) and thus in the inhibition of hair follicle growth, resulting in anagen phase shortening. All these mechanisms result in the formation of vellus or miniature hair together, resulting in total loss of hair if this stimulus persists $(5,12)$.

Treatment methods are limited to FDA-approved minoxidil and finasteride (single or combined use) and hair transplantation. They have various side effects such as hypertrichosis, birth defects in children when given to women of childbearing age, decreased libido and prolonged impotence. Low patient compliance and satisfaction, topical and systemic side effects have led to the search for new treatment options $(1,13)$. Therefore, PRP, which has been shown to be effective in many areas other than plastic surgery and has very few side effects, has attracted attention. Case reports and several patient series have been published on the use of PRP in androgenetic alopecia especially after 2012, and there has been a significant increase in these studies especially in the last two years. The basic idea behind PRP injection is to offer high concentration GFs in the scalp hoping to stimulate hair growth (6).

PRP GFs stimulate the growth of existing miniature hair follicles without interfering with the hormonal pathways of AGA, thus

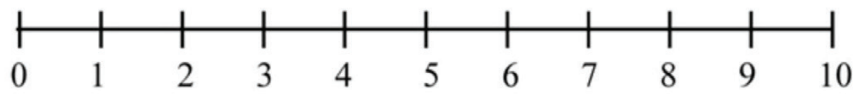

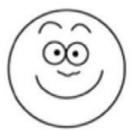

0

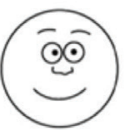

2

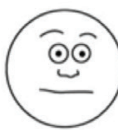

4

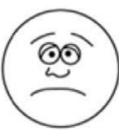

6

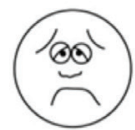

8

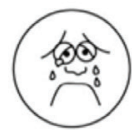

10
Figure 1. Patient satisfaction scale

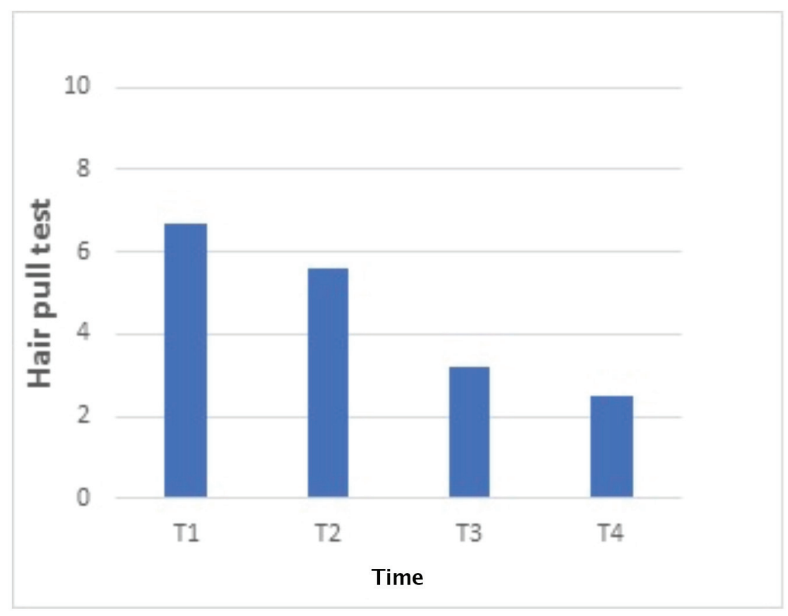

Figure 2. Mean number of hair loss after hair pull test emphasizing the importance of combined therapies to achieve optimal results. GFs are known to activate the proliferative phase, differentiation of hair and stem cells and produce new follicular units. PRP increases cell survival by prolonging the anagen phase of the hair growth cycle and inhibiting apoptosis through increased expression of fibroblast growth factor (FGF7). It has also been shown to increase perifollicular vascular plexus by an increase in vascular endothelial growth factor and platelet derived growth factor levels with angiogenic potential. PRP-induced activation of anti-apoptotic regulators such as BCl2 protein and Akt signal also prolongs the survival of dermal papilla cells during the hair cycle $(6,7,14)$. Therefore, it is a powerful tool for the treatment of androgenetic alopecia.

In our study, PRP was prepared by a single centrifuge method where the blood cells were manually separated into layers and the kit was not used. In our PRP preparation protocol that supports the study of Perez et al. (15), optimum platelet concentration was achieved with low centrifugation time at high acceleration force. Thus, the proliferation of dermal papillary cells is increased and apoptosis is prevented by effectively prepared PRP.

Patients with low-grade alopecia (according to the NorwoodHamilton and Ludwig scale) had better results than those with advanced alopecia. In our study, hair pull test was found to be positive in $86 \%$ of the patients at the $6^{\text {th }}$ month after 3 sessions. This result is comparable to the results of Khatu et al.

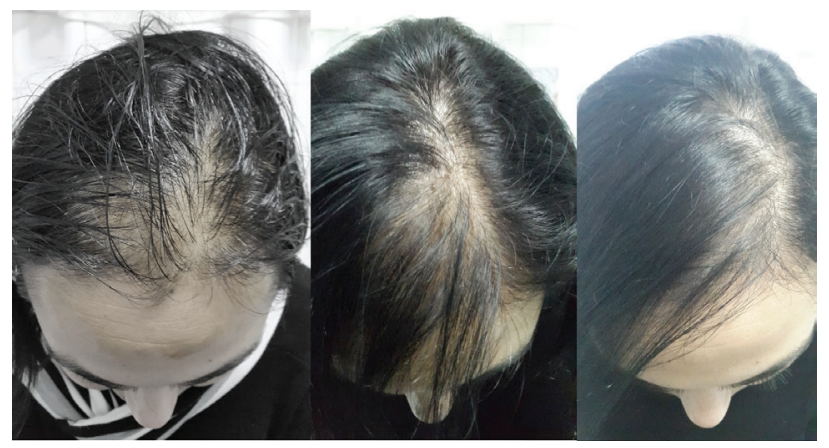

Figure 3. Baseline, $1^{\text {st }}$ month and $6^{\text {th }}$ month photographs of a 45 -yearold female patient after 3 sessions

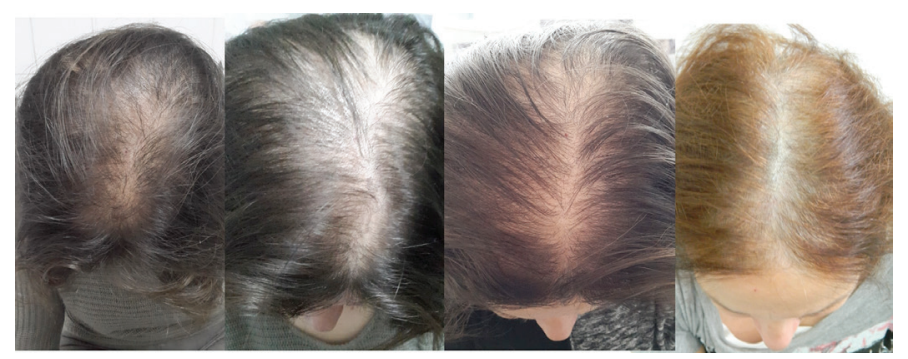

Figure 4. Baseline, $1^{\text {st }}$ month and $6^{\text {th }}$ month photographs of a 51-yearold female patient 
(6) reporting negative hair pull test in $81 \%$ of patients after 4 sessions with an interval of 2 weeks and the results of Besti et al. (16) reporting negative hair pull test in all patients (after the $3^{\text {rd }}$ session) after 5 sessions with an interval of 2 weeks. In addition, Gkini et al. (4) reported negative hair pull test after 3 PRP sessions with an interval of 3 weeks, but they emphasized an increase in the number of hair loss in the 6th month follow-up and applied a booster at $6^{\text {th }}$ months.

The hair evaluation methods we used in our study were not objective methods, but, before each treatment and after each session, it was standardized for each patient. Although hair pull test was carried out in a standard fashion by two evaluators, it is a subjective assessment. Macroscopic photographs give an overview of hair growth, quality and hair density. The TrichoScan assessment, which is a more objective method that should be applied to a shaved part of the patient's scalp, could not be performed, as most patients, especially women, did not consent its application. The VAS score is a subjective method, as it is filled by the patients. However, a suitable method for measuring hair growth over time is not available in a repeatable, economic and non-invasive manner, and these methods have provided a relatively reasonable assessment of the results after treatment. There are some limitations in this study such as small sample size and short follow-up time to assess long-term efficacy of treatment. Therefore, further studies with longer follow-up periods and larger sample groups are needed.

\section{CONCLUSION}

In conclusion, PRP injection, which provides optimal platelet concentration with an effective and easy preparation protocol without using commercial kit, is a simple, low cost and satisfactory treatment option in androgenetic alopecia.

\section{Ethics}

Ethics Committee Approval: Retrospective study.

Informed Consent: Informed consent forms were signed by all patients.

Peer-review: Externally peer-reviewed.

\section{Author Contributions}

Surgical and Medical Practices: K.Ö., Ö.Ç., Concept: K.Ö., Ö.Ç., Design: K.Ö., Ö.Ç., Data Collection or Processing: K.Ö., Ö.Ç., Analysis or Interpretation: K.Ö., Ö.Ç., Literature Search: K.Ö., Ö.Ç., Writing: K.Ö., Ö.Ç.

Conflict of Interest: The authors have no conflicts of interest to declared.
Financial Disclosure: The authors declared that this study has received no financial support.

\section{REFERENCES}

1. Garg S, Manchanda S. Platelet-rich plasma-an 'Elixir' for treatment of alopecia: personal experience on 117 patients with review of literature. Stem Cell Investig 2017;4:64.

2. Starace M, Alessandrini A, D'Acunto C, Melandri D, Bruni F, Patrizi A, et al Platelet-rich plasma on female androgenetic alopecia: Tested on 10 patients. J Cosmet Dermatol 2019;18:59-64.

3. Ayatollahi A, Hosseini H, Shahdi M, AhmadNasrollahi S, NassiriKashani M, Yadangi S, et al. Platelet-rich Plasma by Single Spin Process in Male Pattern Androgenetic Alopecia: Is it an Effective Treatment? Indian Dermatol Online J 2017;8:460-4.

4. Gkini MA, Kouskoukis AE, Tripsianis G, Rigopoulos D, Kouskoukis K. Study of Platelet-Rich Plasma Injections in the Treatment of Androgenetic Alopecia Through an One-Year Period. J Cutan Aesthet Surg 2014;7:213-9.

5. Ferrando J, García-García SC, González-de-Cossío AC, Bou L, Navarra E. A Proposal of an Effective Platelet-rich Plasma Protocol for the Treatment of Androgenetic Alopecia. Int J Trichology 2017;9:165-70.

6. Khatu SS, More YE, Gokhale NR, Chavhan DC, Bendsure N. Platelet-Rich Plasma in Androgenic Alopecia: Myth or an Effective Tool. J Cutan Aesthet Surg 2014;7:107-10.

7. Gentile P, Garcovich S, Bielli A, Scioli MG, Orlandi A, Cervelli V. The Effect of Platelet-Rich Plasma in Hair Regrowth: A Randomized Placebo-Controlled Trial. Stem Cells Transl Med 2015;4:1317-23.

8. Torkamani N, Rufaut NW, Jones L, Sinclair R. Destruction of the arrector pili muscle and fat infiltration in androgenic alopecia. $\mathrm{Br} J$ Dermatol 2014;170:1291-8.

9. Yazdabadi A, Whiting D, Rufaut NW, Sinclair R. Miniaturized hairs maintain contact with the arrector pili muscle in alopecia areata but not in androgenetic alopecia: A model for reversible miniaturization and potential for hair regrowth. Int J Trichology 2012;4:154-7.

10. Torkamani N, Rufaut NW, Jones L, Sinclair RD. Beyond goosebumps: Does the arrector pili muscle have a role in hair loss? Int J Trichology 2014;6;88-94.

11. Dohan Ehrenfest DM, Rasmusson L, Albrektsson T. Classification of platelet concentrates: From pure platelet-rich plasma (P-PRP) to leucocyte- and platelet-rich fibrin (L-PRF) Trends Biotechnol 2009; 27:158-67.

12. Li ZJ, Choi HI, Choi DK, Sohn KC, Im M, Seo YJ, et al. Autologous platelet-rich plasma: A potential therapeutic tool for promoting hair growth. Dermatol Surg 2012;38:1040-6.

13. Jha AK, Udayan UK, Roy PK, Amar AKJ, Chaudhary RKP. Original article: Platelet-rich plasma with microneedling in androgenetic alopecia along with dermoscopic pre- and post- treatment evaluation. J Cosmet Dermatol 2018;17:313-8.

14. Katsuoka K, Schell H, Wessel B, Hornstein OP. Effects of epidermal growth factor, fibroblast growth factor, minoxidil and hydrocortisone on growth kinetics in human hair bulb papilla cells and root sheath fibroblasts cultured in vitro. Arch Dermatol Res 1987;279:247-50.

15. Perez AGM, Lichy R, Lana JFSD, Rodrigues AA, Luzo AC, Belangero WD, et al. Prediction and modulation of platelet recovery by discontinuous centrifugation of whole blood for the preparation of pure platelet-rich plasma. Biores Open Access 2013;2:307-14.

16. Besti EE, Germain E, Kalbermatten DF, Tremp M, Emmenegger V. Plateletrich plasma injection is effective and safe for the treatment of alopecia. Eur J Plast Surg 2013;36:407-12. 